\title{
Reconstruction of Wavefront Aberration of 100-TW Ti:sapphire Laser Pulse Using Phase Retrieval Method
}

\author{
Tae Moon Jeong ${ }^{1 *}$, Chul Min Kim¹, Do-Kyeong Ko ${ }^{1,2}$, and Jongmin Lee ${ }^{1 \dagger}$ \\ ${ }^{1}$ Advanced Photonics Research Institute, GIST, 1 Oryong-dong, Buk-gu, Gwangju 500-712, Korea \\ ${ }^{2}$ School of Photon Science and Technology, GIST, 1 Oryong-dong, Bok-gu, Gwangju 500-712, Korea
}

(Received July 10, 2008 : accepted September 8, 2008)

\begin{abstract}
A phase retrieval method using an error reduction algorithm is developed for reconstructing a wavefront aberration of an 100-TW Ti:sapphire laser pulse from the measurement of a focal spot. The phase retrieval method can successfully reconstruct a wavefront aberration of a 100-TW Ti:sapphire laser pulse, and the reconstructed wavefront aberration shows a good agreement with the wavefront aberration measured with a wavefront sensor. The effect of the dynamic range and the intensity noise on the reconstruction is also investigated in reconstructing a wavefront aberration of an 100-TW Ti:sapphire laser pulse.
\end{abstract}

Keywords: phase retrieval; wavefront aberration; ultrashort high power laser

OCIS codes : (100.5070) Phase retrieval ; (080.1010) Aberrations ; (140.3590) Lasers, titanium ; (140.3295) Laser beam characterization

\section{INTRODUCTION}

As an ultrashort high power laser system becomes popular, the focal spot of a laser pulse becomes important in the laser-plasma interaction study $[1,2]$. The focal spot is mostly determined by the spatial phase profile (called wavefront aberration) over the entire laser pulse. Thus, the measurement and the correction of a wavefront aberration of a laser pulse is important for improving the quality of the focal spot. An adaptive optics (AO) system [3] that consists of a wavefront sensing device $[4,5]$ and a deformable mirror $[6,7]$ measures and corrects a wavefront aberration of a laser pulse, and eventually delivers a nearly diffraction-limited focal spot to a target. However, in many cases, a nearly diffraction-limited focal spot becomes worse as time goes by. This is partly due to the discrepancy between the measured and the real wavefront aberrations. The discrepancy is caused by the change of the propagation direction of a laser pulse, locating the laser pulse in different locations in the measurement system. The evacuation of vacuum chambers and the daily movement of the laser beam propagation direction cause the change of the propagation direction. Unfortunately,

*Corresponding author: jeongtm@apri.gist.ac.kr

${ }^{\dagger}$ Corresponding author: leejm@gist.ac.kr if some parts of the wavefront measurement system are placed in a vacuum chamber, the realignment of the wavefront measurement system is limited during the laser-plasma interaction experiment.

In that circumstance, a phase retrieval method [8] can be an alternative to determine a wavefront aberration of a laser pulse. The phase retrieval method is used to reconstruct amplitude and phase of an unknown object in the fields of wavefront sensing $[9,10]$ and X-ray diffraction microscopy [11]. The phase retrieval method utilizes a focal spot and an intensity distribution of a laser pulse to determine a wavefront of a laser pulse. Iterative Fourier transform algorithm (IFTA) is used to achieve an acceptable solution for a wavefront from the phase retrieval method. For the application of the phase retrieval method to the laser system, Ivanov et al. [12] demonstrated the reconstruction for a laser beam through the phase retrieval method, and later Matsuoka et al. [13] demonstrated the wavefront reconstruction for a terawatt-class CPA Ti:sapphire laser system. Because of the ability of reconstructing a wavefront aberration from the measurement of the Fourier intensity, the phase retrieval method can be used to determine, through a simple measurement, a wavefront aberration of an ultrashort high power laser pulse having a large beam size.

In this paper, we describe a phase retrieval method 
for reconstructing a wavefront aberration and demonstrate the reconstruction of a wavefront aberration of an 100TW CPA Ti:sapphire laser pulse. In sect. 2, a phase retrieval method for reconstructing a wavefront aberration of the 100-TW Ti:sapphire laser pulse is described. The validity of the phase retrieval method is examined by reconstructing a wavefront aberration from the point spread function (PSF) and the beam intensity profile. The effect of the dynamic range and the intensity noise is also investigated for the application of the phase retrieval method to the wavefront reconstruction. In sect. 3 , the reconstruction results for a wavefront aberration of the 100-TW Ti:sapphire laser pulses are discussed. Two different shapes of wavefront aberrations are applied to the 100-TW laser pulse, and reconstructed from the measurement of a focal spot and a beam intensity profile. The reconstructed wavefront aberration shows a good agreement with a wavefront aberration measured with a wavefront sensor. The final conclusion is given in Sect. 4 .

\section{PHASE RETRIEVAL METHOD FOR RECONSTRUCTING WAVEFRONT AVERRATION}

A phase retrieval method using an error reduction algorithm was developed for the reconstruction of a wavefront aberration of the 100-TW Ti:sapphire laser pulse. Two laser-beam intensities in the pupil and the Fourier planes were used as inputs for phase retrieval. Figure 1 describes the phase retrieval method for reconstructing a wavefront aberration from the measurement of two laser-beam intensities. As shown in Fig. 1, the square root (magnitude) of the laser-beam intensity in the pupil plane $\left(\sqrt{I_{\text {pupil }}\left(x_{1}, y_{1}\right)}\right)$ is Fourier transformed with an initial guess for the wavefront aberration $\left(W_{\text {initial }}\left(x_{1}, y_{1}\right)\right)$ to obtain a focal spot in the Fourier plane. The initial guess for the wavefront aberration was obtained by performing the inverse Fourier transform to the square root (magnitude) of the measured intensity distribution (focal spot) in the Fourier plane. Then, the magnitude of the calculated focal spot $\left(\sqrt{I_{n}^{\prime}\left(x_{2}, y_{2}\right)}\right)$ was replaced with the square root (magnitude) of the focal spot $\left(\sqrt{I_{\text {Fourier }}\left(x_{2}, y_{2}\right)}\right)$, still leaving the phase data $\left(\phi_{n}\left(x_{2}, y_{2}\right)\right)$ the same. By performing the inverse Fourier transform to an electric field obtained with the magnitude of the focal spot and the phase data, a new electric field $\left(\sqrt{I_{n+1}\left(x_{1}, y_{1}\right)} \exp \left\{i k W_{n+1}\left(x_{1}, y_{1}\right)\right\}\right)$ was obtained in the pupil plane. The magnitude of the new electric field $\left(\sqrt{I_{n+1}\left(x_{1}, y_{1}\right)}\right)$ was again replaced with the magnitude of the measured beam intensity in the pupil plane $\left(\sqrt{I_{\text {pupil }}\left(x_{1}, y_{1}\right)}\right)$. The Fourier and the inverse Fourier transforms were repeatedly performed to achieve an acceptable solution with a convergence. Two error metrics in the pupil and the Fourier planes defined in ref. [8] were calculated and recorded during the iteration. The reconstructed wavefront aberration was also expressed by the Zernike polynomial representation up to the fifth order. A double-index scheme in the OSA/ANSI standard was used to label the Zernike coefficients [14].

The phase retrieval method was tested with a laser beam profile and a test wavefront aberration of an 100TW laser pulse. The beam intensity in the Fourier plane (focal spot) was obtained by calculating a point spread function (PSF) with the measured intensity and wavefront aberration profiles. The beam size of an incident laser pulse was assumed to be $71 \mathrm{~mm}$ and the intensity distribution in the pupil plane was divided into 120-by-120 pixels. In the wavefront reconstruction, the intensity

$$
W_{1}\left(x_{1}, y_{1}\right)=W_{\text {initial }}\left(x_{1}, y_{1}\right)
$$

\section{for a certain $n$-th iteration}

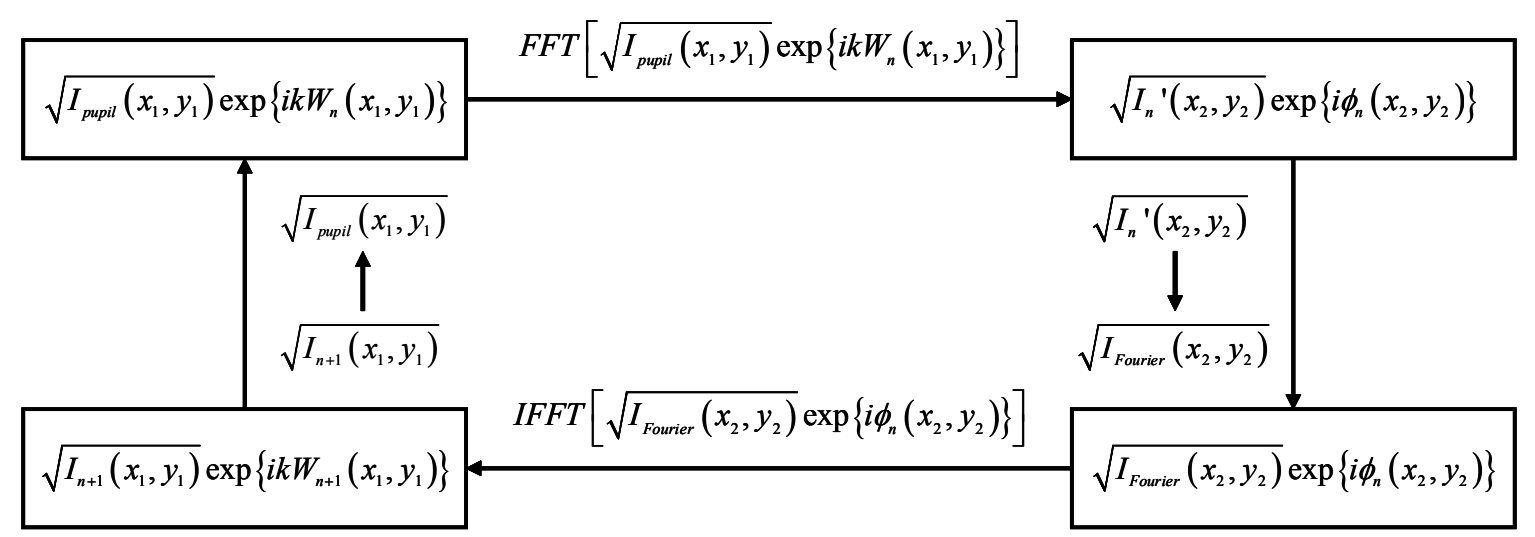

FIG. 1. Phase retrieval method using the error reduction algorithm for reconstructing the wavefront aberration of the 100-TW CPA Ti:sapphire laser pulse. 


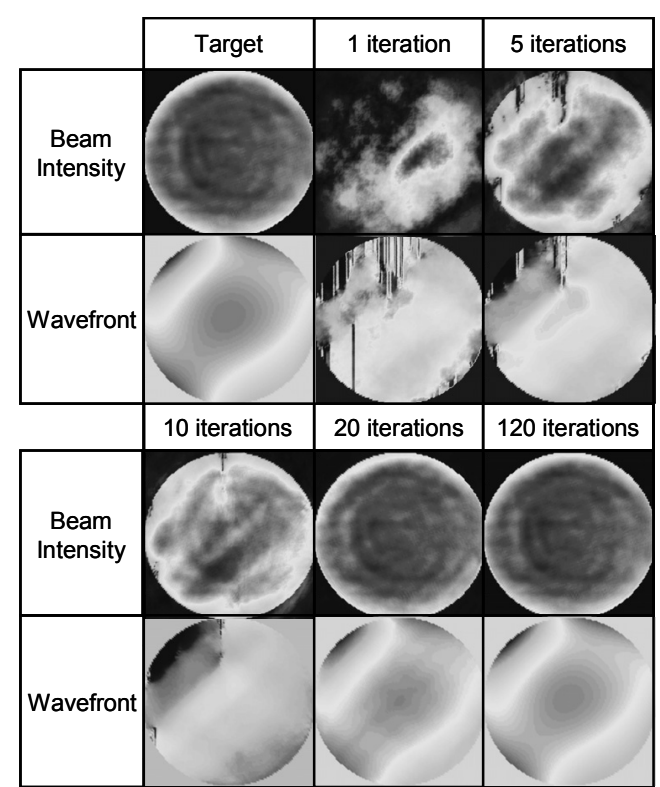

(a)

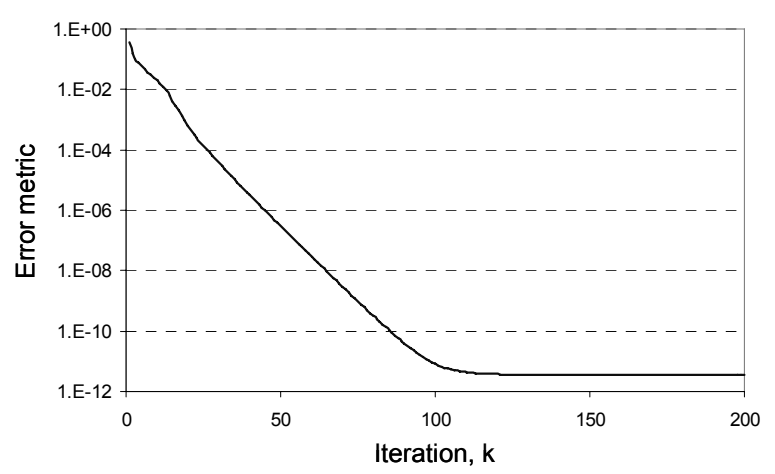

(b)

FIG. 2. (a) Reconstructed beam intensity and wavefront aberration of the 100-TW CPA Ti:sapphire laser pulse with respect to the iteration number. (b) Decrease of the error metric in the pupil plane with respect to the iteration number.

outside the beam size was set to zero for the constraint condition in the pupil plane. The Fourier plane was divided into 240 -by-240 or 480 -by-480 pixels by assuming the use of focusing lenses having focal lengths of about 0.9 and $1.8 \mathrm{~m}$, respectively. A zero padding method was used to obtain 480-by-480 pixels. However, calculations with 240-by-240 and 480-by-480 pixels in the Fourier plane show almost identical reconstruction results for the test wavefront. Figure 2(a) shows the reconstructed intensity profiles and the wavefront aberration maps as a function of iteration. As shown in Fig. 2(a), the intensity and the wavefront profiles are successfully reconstructed as the number of iteration increases. In this case, the pupil plane error metric converged in 120 iterations (See Fig. 2(b)). To quantitatively examine the accuracy of the reconstructed wavefront, the difference between individual Zernike coefficients for the

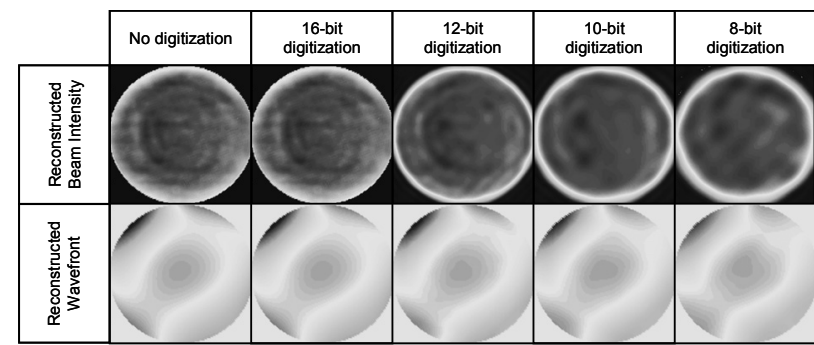

(a)

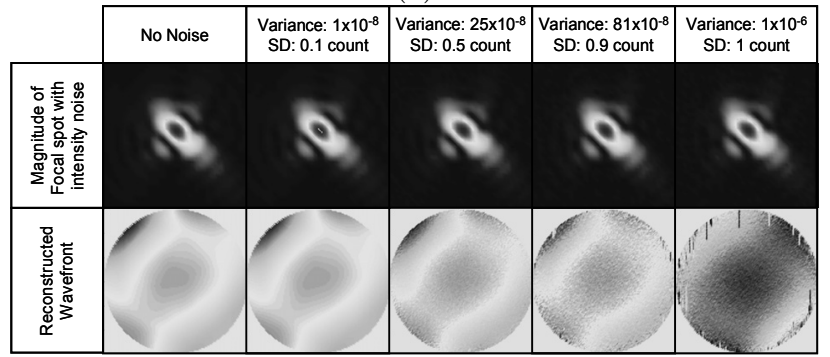

(b)

FIG. 3. (a) Reconstructed beam intensity and wavefront aberration with respect to the dynamic range of the CCD camera. (b) Reconstructed wavefront aberrations with the increase of Guassian white noise. The standard deviation (SD) in the figure was calculated by assuming the use of the 10-bit CCD camera.

test and the reconstructed wavefront aberrations were investigated. The accuracy was expressed in the rms (root-mean-square) value of the difference of individual Zernike coefficients. The reconstructed wavefront was almost identical to the test wavefront, yielding the rms value of $0.001 \mu \mathrm{m}$ after 200 iterations.

Because a CCD camera has the finite intensity resolution (dynamic range), the dependence of the reconstruction performance on the intensity resolution was investigated. In the calculations, the use of 8-, 10-, 12-, and 16-bit CCD cameras was assumed for the dynamic range. Figure 3(a) shows the reconstructed intensities and wavefront aberration profiles obtained by assuming the use of $8-, 10-, 12$, and 16-bit CCD cameras. The reconstruction for the beam intensity became worse with the decrease of the dynamic range. However, the reconstruction for the wavefront aberration was not sensitive to the dynamic range of a CCD camera. The rms error for the individual Zernike coefficients between the original and the reconstructed wavefront aberration was only $0.012 \mu \mathrm{m}$ when the use of an 8-bit CCD camera was assumed. Thus, it can be concluded that the use of a 10-bit CCD camera is good enough for the wavefront reconstruction.

Another factor to affect the wavefront reconstruction is the intensity noise that can be recorded together with the intensity data in the Fourier plane. We also investigated the effect of the intensity noise on the wavefront reconstruction. A Gaussian white noise was assumed as the intensity noise recorded with the intensity data in 
the Fourier plane. The "imnoise" function in the Matlab software was used to generate a Gaussian white noise in the Fourier plane. To examine the effect of the intensity noise on the wavefront reconstruction, the wavefront reconstruction was performed with the increase of the variance or the standard deviation (SD) of the noise level in the Fourier intensity. In the calculation, the variance increased from $1 \times 10^{-8}$ to $1 \times 10^{-6}$ with the normalized intensity data in the Fourier plane. This means that the standard deviation of the noise intensity increased from 0.1 to 1 count with a 10-bit CCD camera. Figure 3(b) shows the reconstructed wavefront aberration maps with the increase of the noise level in the Fourier intensity. As shown in Fig. 3(b), the reconstruction performance became worse as the Gaussian white noise in the Fourier intensity increased. Above the variance of $1 \times 10^{-6}$, the wavefront aberration map was not well reconstructed from the Fourier intensity. This calculation shows that the Gaussian white noise should be well controlled in recording the Fourier intensity.

\section{WAVEFRONT RECONSTRUCTION OF AN 100-TW TI:SAPPHIRE LASER PULSE}

The phase retrieval method was applied to the wavefront reconstruction of the 100-TW Ti:sapphire laser system at the Advanced Photonics Research Institute (APRI). A compressed 100-TW Ti:sapphire laser pulse passes through an adaptive optics (AO) system for the correction of the wavefront aberration. Detailed features of the 100-TW Ti:sapphire laser system and the performance of the AO system are described elsewhere $[3,15,16]$. Figure 4 shows an optical layout for recording laser-beam intensities that are used as inputs for the wavefront reconstruction. As shown in Fig. 4, the transmitted laser pulse from the full reflector (M1, reflectance: $>99.9 \%$ ) passes through a window attached on a compression chamber and is focused by an achromatic lens having a focal length of $1.5 \mathrm{~m}$. A folding mirror (M2) was placed to turn the beam direction to a 10-bit CCD camera having

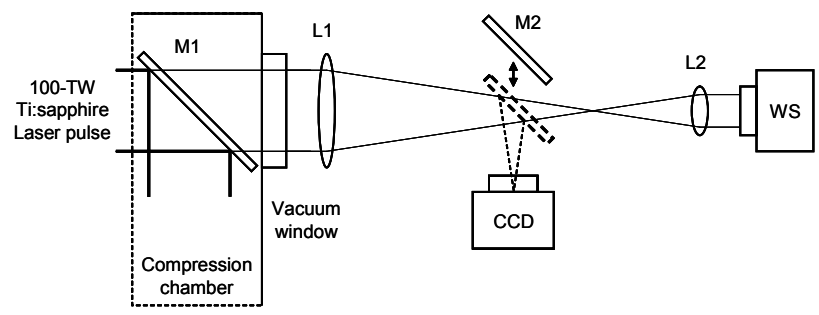

FIG. 4. Optical layout for measuring intensity distribution (focal spot) of the 100-TW CPA Ti:sapphire laser pulse in the Fourier plane. The focal spot was measured with the CCD camera. The reconstructed wavefront aberration was compared to the wavefront aberration measured with the wavefront sensor (WS in the figure). a pixel size of $5.2 \mu \mathrm{m}$. By taking the folding mirror out, the transmitted laser pulse propagates to an achromatic lens having a focal length of $7.5 \mathrm{~cm}$, and is collimated after the lens. The collimated laser pulse is incident on a wavefront sensor (Phasics, SID-4) that can measure the wavefront aberration and the intensity profile of the laser pulse. The measured intensity profile is used as input data for the wavefront reconstruction. The measured wavefront aberration was compared to the wavefront aberration reconstructed from the focal spot measurement.

Because of the broad spectrum of an ultrashort high power laser pulse, the chromatic aberration can be induced in the ultrashort high power laser pulse and can deform the focal spot. The temporal and spatial deformation of the focal spot by the chromatic aberration was described elsewhere [16]. In addition, the broad spectrum of an ultrashort high power laser pulse may induce the angular dispersion (spatial chirping) even if compression gratings are just slightly misaligned. The angular dispersion results in the elongation of the focal spot. The deformation and the elongation of the focal spot are not appropriate for the wavefront reconstruction. In the focal spot measurement, a bandpass filter having a bandwidth of $10 \mathrm{~nm}$ at $800 \mathrm{~nm}$ was placed in front of the 10-bit CCD camera to minimize the effect of the broad spectrum. With the bandpass filter, the change in the Zernike coefficient for defocus $\left(\mathrm{Z}_{2}^{0}\right)$ varied only about $0.05 \mu \mathrm{m}$ in the range of 795 to $805 \mathrm{~nm}$.

Now, we describe the reconstruction result of a wavefront aberration for the 100-TW Ti:sapphire laser pulse. First, the wavefront aberration of the 100-TW Ti:sapphire laser pulse was compensated for with a deformable mirror in the AO system. Then, a certain aberration mode in the Zernike representation, such as astigmatism $\left(\mathrm{Z}_{2}{ }^{2}\right.$ or $\mathrm{Z}_{2}^{-2}$ ), coma $\left(\mathrm{Z}_{3}{ }^{1}\right.$ or $\left.\mathrm{Z}_{3}^{-1}\right)$, and spherical aberration $\left(\mathrm{Z}_{4}{ }^{0}\right)$, was applied to the laser pulse. A bimorph deformable mirror having 32 channels was used to correct and generate a wavefront aberration in the laser pulse. Figure 5 (a) shows the wavefront aberration map measured with the wavefront sensor when astigmatism $\left(\mathrm{Z}_{2}^{-2}\right)$ and coma $\left(Z_{3}{ }^{1}\right)$ aberrations were applied. The wavefront aberration map was reconstructed from the focal spot recorded with astigmatism $\left(\mathrm{Z}_{2}^{-2}\right)$ and coma $\left(\mathrm{Z}_{3}{ }^{1}\right)$ aberrations and the intensity profile of the laser pulse. Figure $5(\mathrm{~b})$ shows the reconstructed wavefront map calculated from the focal spot and the intensity profile. In this case, 200 iterative calculations were performed to obtain the reconstructed wavefront map. As shown in Fig. 5(a) and 5(b), two wavefront aberration maps are very similar to each other. The individual Zernike coefficients are also shown in figure $5(\mathrm{c})$ for the examination of the similarity between two wavefront aberration maps. Error bars mean the standard deviations of the five successive measurements. As shown in Fig. 5(c), astigmatism $\left(\mathrm{Z}_{2}^{-2}\right)$ and coma $\left(\mathrm{Z}_{3}{ }^{1}\right)$ aberration modes were well reconstructed by the 


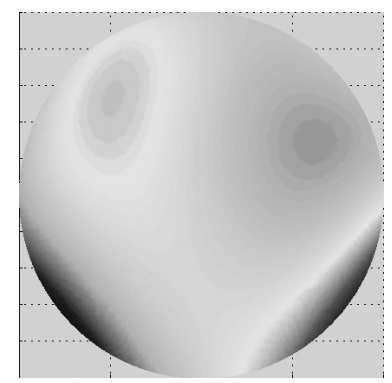

(a)

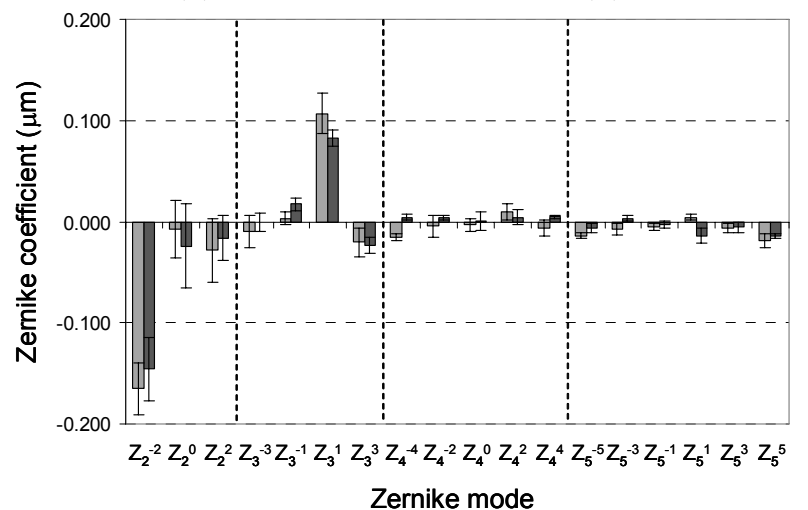

(c)

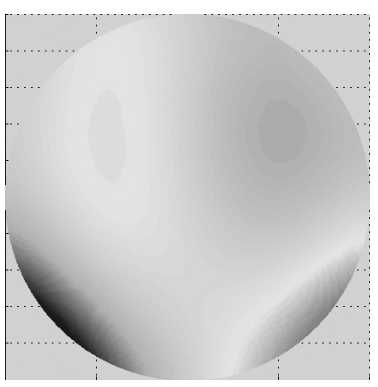

(b)
FIG. 5. (a) Wavefront aberration map measured with the wavefront sensor. (b) Wavefront aberration map reconstructed with the phase retrieval method. (c) Comparison the reconstructed wavefront aberration map. Error bars mean the standard deviation of the measurement. Astigmatism $\left(\mathrm{Z}_{2}^{-2}\right)$ and coma $\left(\mathrm{Z}_{3}^{1}\right)$ were well reconstructed by the phase retrieval method.

phase retrieval algorithm. The accuracy defined by the rms value of the difference between Zernike coefficients for the measured and the reconstructed wavefront was $0.05 \mu \mathrm{m}$.

Figure 6 shows another reconstruction results for the 100-TW Ti:sapphire laser pulse when astigmatism $\left(\mathrm{Z}_{2}^{-2}\right)$, coma $\left(\mathrm{Z}_{3}^{1}\right)$, and spherical aberration $\left(\mathrm{Z}_{4}^{0}\right)$ were applied. Figure 6(a) and 6(b) show the measured and the reconstructed wavefront aberration maps. Again, astigmatism, coma, and spherical aberration were well reconstructed by the phase retrieval method (See Fig. 6(c)). The accuracy defined by the rms value was about $0.06 \mu \mathrm{m}$ in this case. From the reconstruction results, it can be concluded that the phase retrieval method using an error reduction algorithm can successfully reconstruct the wavefront aberration of the 100-TW Ti:sapphire laser pulse.

\section{CONCLUSIONS}

The phase retrieval method had been developed for reconstructing a wavefront aberration of the 100-TW Ti:sapphire laser pulse and its validity was examined by reconstructing a wavefront aberration of the 100-TW

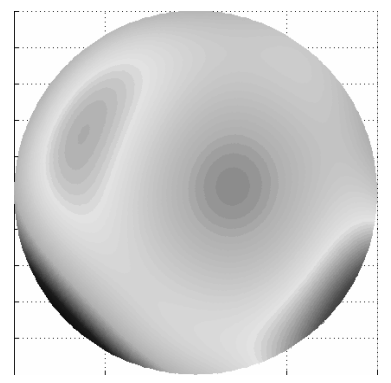

(a)

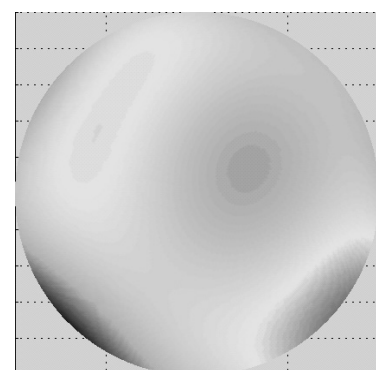

(b)

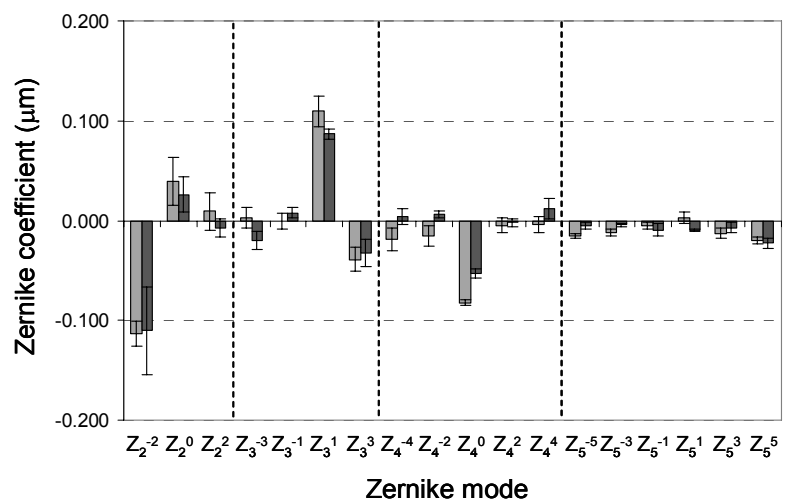

(c)
FIG. 6. (a) Wavefront aberration map measured with the wavefront sensor. (b) Wavefront aberration map reconstructed with the phase retrieval method. (c) Comparison of individual Zernike coefficients between the measured and the reconstructed wavefront aberration map. Astigmatism $\left(\mathrm{Z}_{2}^{-2}\right)$, coma $\left(\mathrm{Z}_{3}{ }^{1}\right)$, and spherical aberration $\left(\mathrm{Z}_{4}^{0}\right)$ were well reconstructed by the phase retrieval method.

Ti:sapphire laser pulse. The intensity distributions in the pupil and the Fourier planes were measured for the wavefront reconstruction of the 100-TW Ti:sapphire laser pulse. The reconstructed wavefront aberration showed a good agreement when it was compared to the aberration measured with a wavefront sensor. The accurate reconstruction of the wavefront aberration using the phase retrieval method also will help to build a low-cost adaptive optics (AO) system for an ultrashort high power laser system.

\section{ACKNOWLEDGMENT}

This work was supported by the Ministry of Knowledge and Economy of Korea through the Ultrashort Quantum Beam Facility Program.

\section{REFERENCES}

[1] N. M. Hafz, I. W. Choi, J. H. Sung, H. T. Kim, K.-H. Hong, T. M. Jeong, T. J. Yu, V. Kulagin, H. Suk, Y.-C. 
Noh, D.-K. Ko, and J. Lee, "Dependence of the electron beam parameters on the stability of laser propagation in a laser wakefield accelerator," Appl. Phys. Lett., vol. 90, 151501, 2007.

[2] N. M. Hafz, T. M. Jeong, I. W. Choi, S. K. Lee, K. H. Pae, V. Kulagin, J. H. Sung, T. J. Yu, K.-H. Hong, T. Hosokai, J. R. Cary, D.-K. Ko, and J. Lee, "Stable generation of $\mathrm{GeV}$-class electron beams from self-guided laser plasma channels," Nature Photonics, vol. 2, pp. 571-577, 2008.

[3] T. M. Jeong, I. W. Choi, N. Hafz, J. H. Sung, D.-K. Ko, and J. Lee, "Wavefront Correction and Customization of Focal Spot of 100 TW Ti:sapphire Laser System," Jpn. J. Appl. Phys., vol. 46, no. 12, pp. 7724-7730, 2007.

[4] T. M. Jeong, D.-K. Ko, and J. Lee, "Method of reconstructing wavefront aberrations by use of Zernike polynomials in radial shearing interferometers," Opt. Lett., vol. 32, no. 3, pp. 232-234, 2007.

[5] T. M. Jeong, M. Menon, and G. Yoon, "Measurement of wave-front aberration in soft contact lenses by use of a Shack-Hartmann wavefront sensor," Appl. Opt., vol. 44, no. 21, pp. 4523-4527, 2005.

[6] J. H. Lee, Y. C. Lee, and H. J. Cheon, "A Circular Bimorph Deformable Mirror for Circular/Annulus/Square Laser Beam Compensation,” J. Opt. Soc. Korea, vol. 10, no. 1, pp. 23-27, 2006.

[7] J. H. Lee, Y. C. Lee, and E. C. Kang, "A Cooled Deformable Bimorph Mirror for a High Power Laser," J. Opt. Soc. Korea, vol. 10, no. 2, pp. 57-62, 2006.

[8] J. R. Fienup, "Phase retrieval algorithms: a comparison," Appl. Opt., vol. 21, no. 15, pp. 2758-2769 1982.
[9] J. N. Ciderquist, J. R. Fienup, C. C. Wackerman, S. R. Robinson, and D. Kryskowski, "Wavefront phase estimation from Fourier intensity measurements," J. Opt. Soc. Am. A, vol. 6, no. 7, pp. 1020-1026, 1989.

[10] F. Roddier and C. Roddier, "Wavefront reconstruction using iterative Fourier transforms,” Appl. Opt., vol. 30, no. 11, pp. 1325-1327, 1991.

[11] J. Miao, P. Charalambous, J. Kirz, and D. Sayre, "Extending the methodology of X-ray crystallography to allow imaging of micrometer-sized non-crystalline specimens," Nature, vol. 400, pp. 342-344, 1999.

[12] V. Yu. Ivanov, V. P. Sivokon, and M. A. Vorontsov, "Phase retrieval from a set of intensity measurements: theory and experiment," J. Opt. Soc. Am. A, vol. 9, no. 9, pp. 1515-1524, 1992.

[13] S. Matsuoka and K. Yamakawa, "Wave-front measurements of terawatt-class ultrashort laser pulses by the Fresnel phase-retrieval method," J. Opt. Soc. Am. B, vol. 17 , no. 4 , pp. 663-667, 2000.

[14] ANSI Z80.28-2004, Methods for reporting optical aberrations of eyes (American National Standards Institute, 2004).

[15] T. M. Jeong, I. W. Choi, J. H. Sung, H. T. Kim, K-H. Hong, T. J. Yu, J. H. Kim, Y.-C. Noh, D.-K. Ko, J. Lee, A. Sagisaka, A. Yogo, and H. Daido, "Measurement of the Electron Density Produced by the Prepulse in an Experiment of High Energy Proton Beam Generation," J. Korean Phys. Soc., vol. 50, no. 1, pp. 34-39, 2007.

[16] T. M. Jeong, D.-K. Ko, and J. Lee, "Deformation of the Focal Spot of an Ultrashort High-Power Laser Pulse due to Chromatic Aberration by a Beam Expander," J. Korean Phys. Soc., vol. 52, no. 6, pp. 1767-1773, 2008. 